REVIEW ARTICLE
A.C. Miracle
S.K. Mukherji

\title{
Conebeam CT of the Head and Neck, Part 2: Clinical Applications
}

\begin{abstract}
SUMMARY: Conebeam x-ray CT (CBCT) is being increasingly used for point-of-service head and neck and dentomaxillofacial imaging. This technique provides relatively high isotropic spatial resolution of osseous structures with a reduced radiation dose compared with conventional CT scans. In this second installment in a 2-part review, the clinical applications in the dentomaxillofacial and head and neck regions will be explored, with particular emphasis on diagnostic imaging of the sinuses, temporal bone, and craniofacial structures. Several controversies surrounding the emergence of CBCT technology will also be addressed.
\end{abstract}

$\mathbf{C}_{\text {o }}$ onebeam CT (CBCT) is an advancement in CT imaging that has begun to emerge as a potentially low-dose crosssectional technique for visualizing bony structures in the head and neck. The physical principles, image quality parameters, and technical limitations relevant to CBCT imaging were discussed in Part 1 of this 2-part series. The second part presented here will highlight the evidence related to CBCT applications in head and neck as well as dentomaxillofacial imaging. Controversial aspects of this technology will also be addressed, including limitations in image quality and its often officebased operational model.

CBCT was first adapted for potential clinical use in 1982 at the Mayo Clinic Biodynamics Research Laboratory. ${ }^{1}$ Initial interest focused primarily on applications in angiography in which soft-tissue resolution could be sacrificed in favor of high temporal and spatial-resolving capabilities. Since that time, several CBCT systems have been developed for use both in the interventional suite and for general applications in CT angiography. ${ }^{2,3}$ Exploration of CBCT technologies for use in radiation therapy guidance began in $1992,{ }^{4,5}$ followed by integration of the first CBCT imaging system into the gantry of a linear accelerator in $1999 .^{6}$

The first CBCT system became commercially available for dentomaxillofacial imaging in 2001 (NewTom QR DVT 9000; Quantitative Radiology, Verona, Italy). Comparatively low dosing requirements and a relatively compact design have also led to intense interest in surgical planning and intraoperative CBCT applications, particularly in the head and neck but also in spinal, thoracic, abdominal, and orthopedic procedures..$^{7-11}$ Diagnostic applications in CT mammography and head and neck imaging are also under evaluation. ${ }^{12-14}$ The technical and clinical considerations pertaining to CBCT imaging in many of these applications have been the subjects of several recent reviews. ${ }^{15-19}$ The recent review by Dörfler et $\mathrm{al}^{16}$ of the neurointerventional applications of CBCT is of particular interest to the field of neuroradiology.

The discussion below will focus on the diagnostic and treatment-planning applications of CBCT in dentomaxillofa-

From the Departments of Radiology (A.C.M., S.K.M.), Otolaryngology (S.K.M.), and Radiation Oncology (S.K.M.), University of Michigan Health System, University Hospital, Ann Arbor, Mich.

Please address correspondence to Aaron Miracle, University of Michigan Medical Center, Department of Radiology, B2B311 UH SPC 5030, 1500 East Medical Center Dr, Ann Arbor, Ml 48109-5030; e-mail: amiracle@med.umich.edu

Indicates open access to non-subscribers at www.ajnr.org

DOI 10.3174/ajnr.A1654 cial and head and neck imaging. Commercially available CBCT systems for dentomaxillofacial imaging include the $\mathrm{CB}$ MercuRay and CB Throne (Hitachi Medical, Kashiwi-shi, Chiba-ken, Japan), 3D Accuitomo products (J. Morita Manufacturing, Kyoto, Japan), and iCAT (Xoran Technologies, Ann Arbor, Mich; and Imaging Sciences International, Hatfield, $\mathrm{Pa}$ ). Similar systems designed for point-of-service head and neck imaging have also recently become available (MiniCAT, Xoran Technologies; 3D Accuitomo and 3D Accuitomo 170, J Morita Manufacturing; ILUMA Cone Beam CT, IMTEC, Ardmore, Okla and GE Healthcare, Chalfont St. Giles, UK).

\section{Dentomaxillofacial Imaging}

Advanced cross-sectional imaging techniques such as CT are used in dentomaxillofacial imaging to solve complex diagnostic and treatment-planning problems, such as those encountered in craniofacial fractures, endosseous dental-implant planning, and orthodontics, among others. With the advent of CBCT technology, cross-sectional imaging that had previously been outsourced to medical CT scanners has begun to take place in dental offices.

Early dedicated CBCT scanners for dental use were characterized by Mozzo et $\mathrm{al}^{20}$ and Arai et $\mathrm{al}^{21}$ in the late 1990s. Since then, more commercial models have become available, inciting research in many fields of dentistry and oral and maxillofacial surgery. To date, multiple ex vivo studies have attempted to establish the ability of CBCT images to accurately reproduce the geometric dimensions of the maxillodental structures and the mandible. ${ }^{22-25}$

A relatively low patient dose for dedicated dentomaxillofacial scans is a potentially attractive feature of CBCT imaging. The dosing characteristics of dentomaxillofacial scanners were discussed in Part 1 of this series. An effective dose in the broad range of 13-498 $\mu \mathrm{Sv}$ can be expected, with most scans falling between 30 and $80 \mu \mathrm{Sv}$, depending on exposure parameters and the selected FOV size. In comparison, standard panoramic radiography delivers $\sim 13.3 \mu \mathrm{Sv}$ and multidetector CT with a similar FOV delivers $\sim 860 \mu \mathrm{Sv} .^{26,27}$ Image quality can vary considerably with dose; images acquired with higher radiation exposure often produce superior image quality.

The discussion below reviews potential CBCT applications in the dentomaxillofacial regions. Most of this research remains preliminary; further prospective and outcomes-based research is required to make informed recommendations on the appropriate use of CBCT in dentomaxillofacial imaging. 


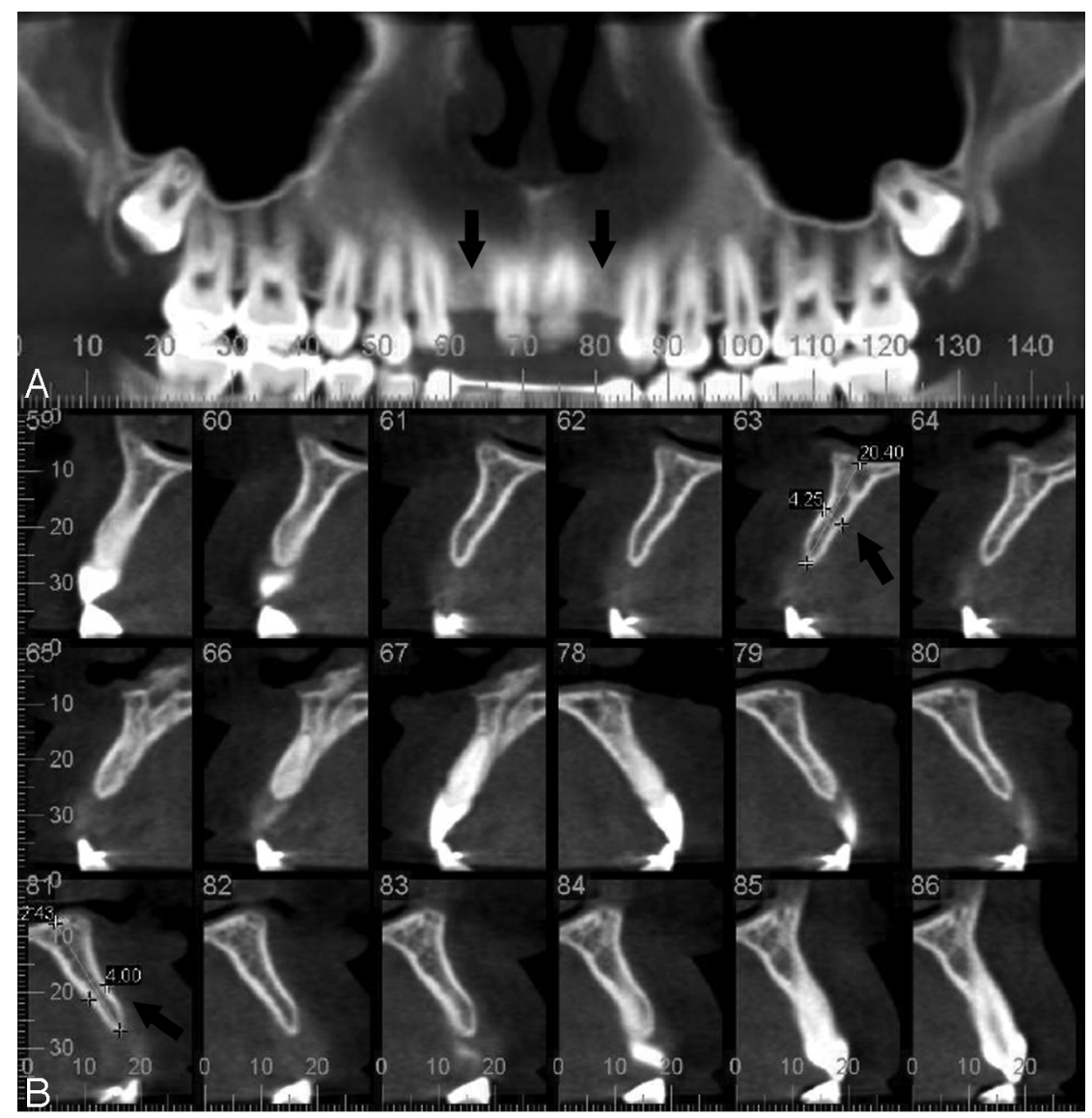

Fig 1. Noncontrast dentomaxillofacial CBCT scan (iCAT) of a patient with congenital absence of the maxillary lateral incisors (0.4-mm pixels, $120 \mathrm{kVp}, 18.66 \mathrm{~mA}$ ). $A$, Reconstructed panoramic view of the maxilla demonstrates bilateral lateral incisor absence (arrows). B, Sequential parasagittal/oblique views through the maxillary alveolar bone demonstrate planned implant locations (arrows).

\section{Implantology}

Cross-sectional imaging techniques can be an invaluable tool during preoperative planning for complicated endosseous dental implantation procedures. ${ }^{28}$ Conventional linear tomography and CT have traditionally been used in presurgical imaging, though the former has overlain ghosting artifacts and the latter has relatively high radiation exposure and cost. ${ }^{29}$

Practitioners have begun using office-based CBCT scanners in preoperative imaging for implant procedures, capitalizing on availability and low dosing requirements. A review by Guerrero et $\mathrm{al}^{30}$ outlines the clinical and technical aspects of $\mathrm{CBCT}$, which have popularized this new technique. Preliminary evidence addresses the ability of CBCT images to characterize mandibular and alveolar bone morphology, as well as to visualize the maxillary sinuses, incisive canal, mandibular canal, and mental foramina, all structures particularly important in surgical planning for dental implantation. ${ }^{29,31,32}$ Several studies have described the 3D geometric accuracy of CBCT imaging in the maxillodental and mandibular regions as well. ${ }^{22-25}$ Examples of CBCT imaging studies for implant planning and visualization of the mandibular canal are presented in Figures 1 and 2 respectively.

\section{Craniofacial Fractures}

Imaging of complex high-contrast bony structural pathology such as craniofacial fractures is a logical application for CBCT. Terakado et $\mathrm{al}^{33}$ reported a case series in 2000 , which included
2 patients with facial trauma for whom СВСТ was used to characterize a mandibular head fracture, dental root fractures, and the displacement of anterior maxillary teeth. Since that time, several additional reports have extolled the low-dose high-resolution properties of CBCT imaging in preoperative characterization of mandibular and orbital floor fractures. ${ }^{34-36}$ In orbital floor fractures, although CBCT can demonstrate orbital content herniation, it lacks the contrast resolution to differentiate the tissue composition of the herniated materials. ${ }^{35}$

The intraoperative uses of C-arm CBCT systems have been evaluated for fractures of the zygomaticomaxillary complex (ZMC), demonstrating the feasibility of CBCT use in surgical navigation, localization of bony fragments, and evaluation of screw anchorage and plate fittings with low levels of metal artifact. ${ }^{37,38}$ These results have been corroborated in a study of postoperative patients with ZMC fractures, though investigators noted that poorly aerated ethmoidal air cells limit the ability of CBCT to visualize the medial orbital wall. ${ }^{39}$ Low bone density in older patients also reduced bony structural definition in their series. Intraoperative efficacy has been evaluated in mandibular fracture fixation as well. ${ }^{40}$

\section{Orthodontics}

Cross-sectional imaging affords overlay-free visualization of structural and anatomic relationships important for addressing many radiologic questions in orthodontics. The current 

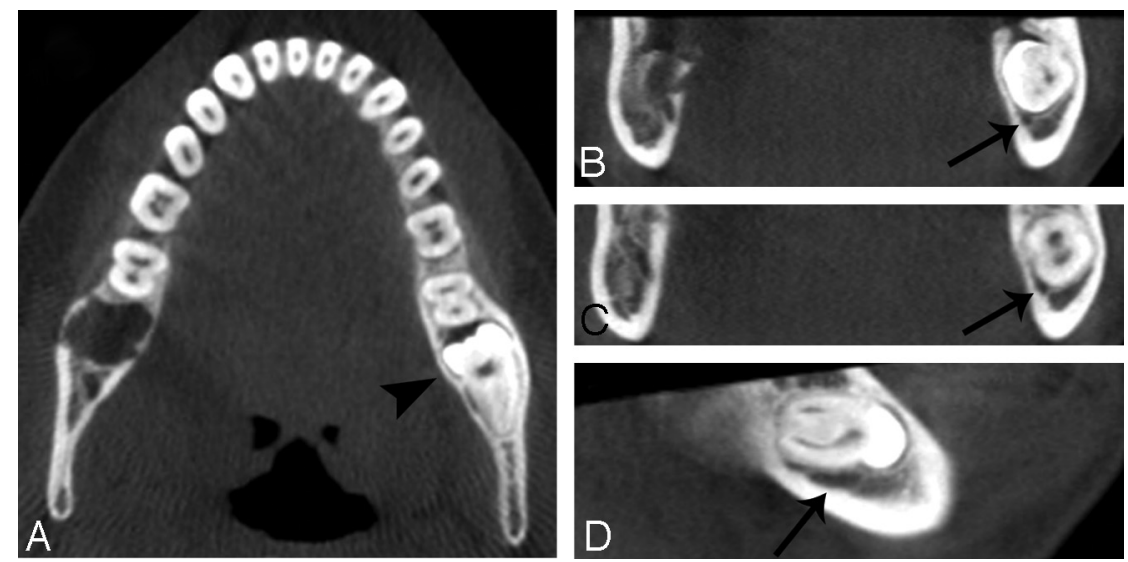

Fig 2. Noncontrast dentomaxillofacial CBCT scan (iCAT) of a patient with an impacted left mandibular third molar (0.4-mm pixels, $120 \mathrm{kVp}, 18.66 \mathrm{~mA})$. A, Axial view through the mandible demonstrates the impacted molar on the left side (arrowhead). $B-D$, Coronal $(B$ and $C$ ) and sagittal $(D)$ views through the mandibular body depict the proximity of the underlying mandibular canal (arrows) to the impacted molar.
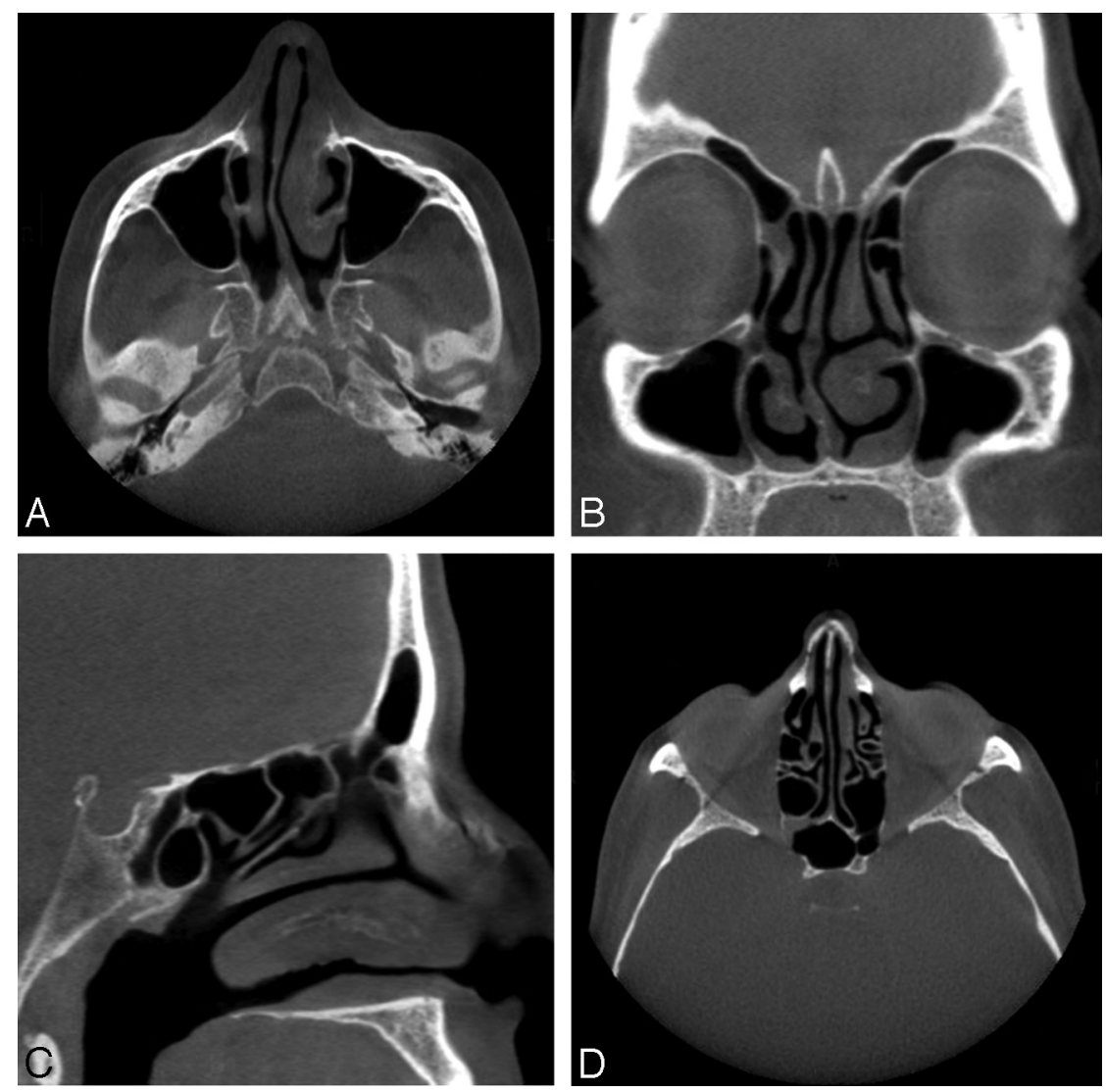

Fig 3. Noncontrast CBCT scan of a 56 -year-old acquired with a sinus protocol ( 40 seconds, 600 frames, 0.4-mm pixels, 120 kVp, $48 \mathrm{~mA}$ ). A, Axial section demonstrates right-sided deviation of the nasal septum and mucosal thickening in the left nasal cavity. $B$, Coronal section redemonstrates mucosal thickening of the left nasal cavity. $C_{r}$ Left paramedian sagittal section. $D$, Axial view of the ethmoid air cells and sphenoid sinus with mild opacification in the region of the right sphenoethmoidal recess.

standard of care for overlay-free imaging in orthodontics is conventional CT. ${ }^{41}$ Low-cost office-based CBCT imaging has recently been explored for orthodontic applications, including assessment of palatal bone thickness, skeletal growth patterns, dental age estimation, upper airway evaluation, and visualization of impacted teeth. ${ }^{42-47}$ Although preliminary results are encouraging, established cross-sectional techniques such as conventional CT provide superior image quality of dental and surrounding structures for advanced orthodontic treatment planning. ${ }^{41}$ Low dosing requirements appear to remain a ben- efit of CBCT when compared with conventional CT, with a routine orthodontic CBCT study delivering an effective dose of $\leq 61.1 \mu \mathrm{Sv}$ compared with $429.7 \mu \mathrm{Sv}$ for multisection CT. ${ }^{48}$ Lateral cephalograms deliver $10.4 \mu \mathrm{Sv}$ in comparison, though without the benefit of 3D structural visualization.

\section{Temporomandibular Joint}

Morphologic changes of the temporomandibular joint (TMJ) as depicted with conventional MR imaging, CT, and radiographic imaging are often useful in diagnosing pathologic 

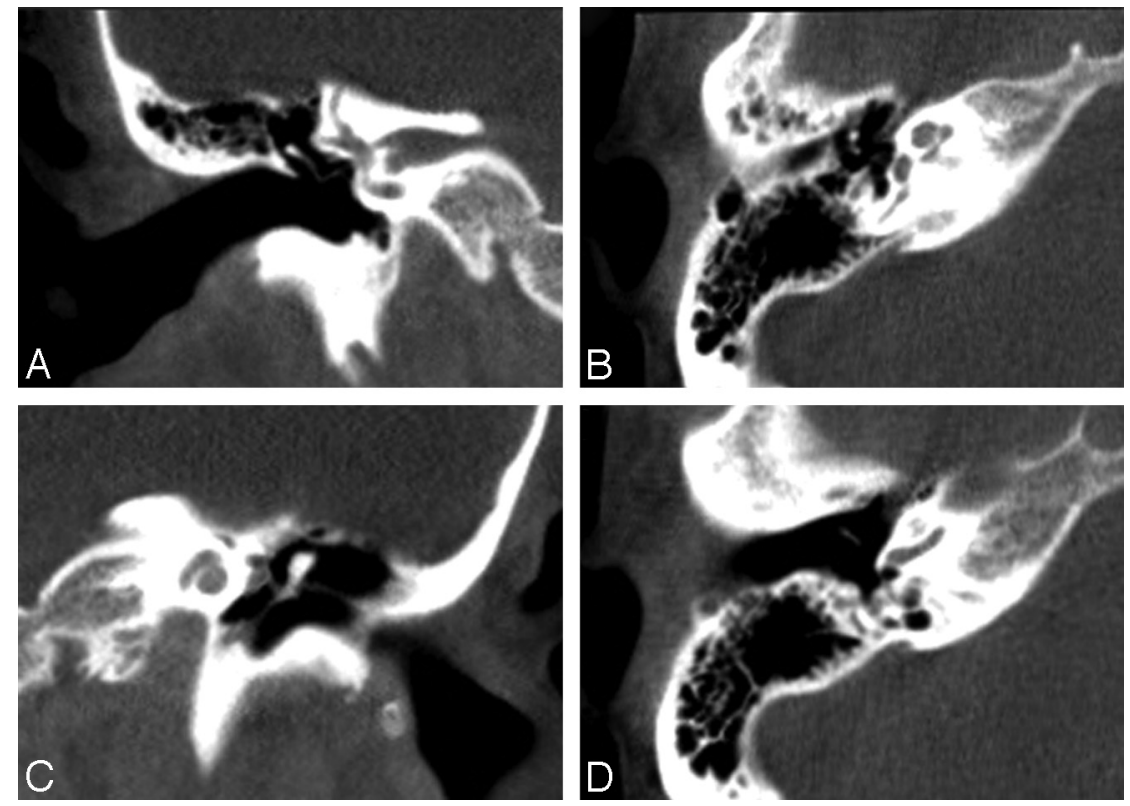

Fig 4. Noncontrast CBCT scan of a 50-year-old acquired with a temporal bone protocol (40 seconds, 600 frames, $0.3-\mathrm{mm}-$ pixels, $125 \mathrm{kVp}, 50.85 \mathrm{~mA}$ ). $A$, Coronal image of the normal right temporal bone demonstrates the vestibulocochlear nerve, body and long limb of the incus, as well as the stapedial neck and crura in the fossa of the oval window. $B$, Axial section at the level of the right mesotympanum demonstrates the head of the malleus, short limb of the incus, and stapedial crura, as well as the cochlear nerve, tensor tympani, and mastoid part of the facial nerve VII. C C Coronal section through the left cochlea demonstrates the modiolus, tympanic part of facial nerve VII, tensor tympani, and malleus. $D$, Axial section through the right mesotympanum at the level of the round window demonstrates the handle of the malleus, base of cochlea, and mastoid portion of the facial nerve VII.
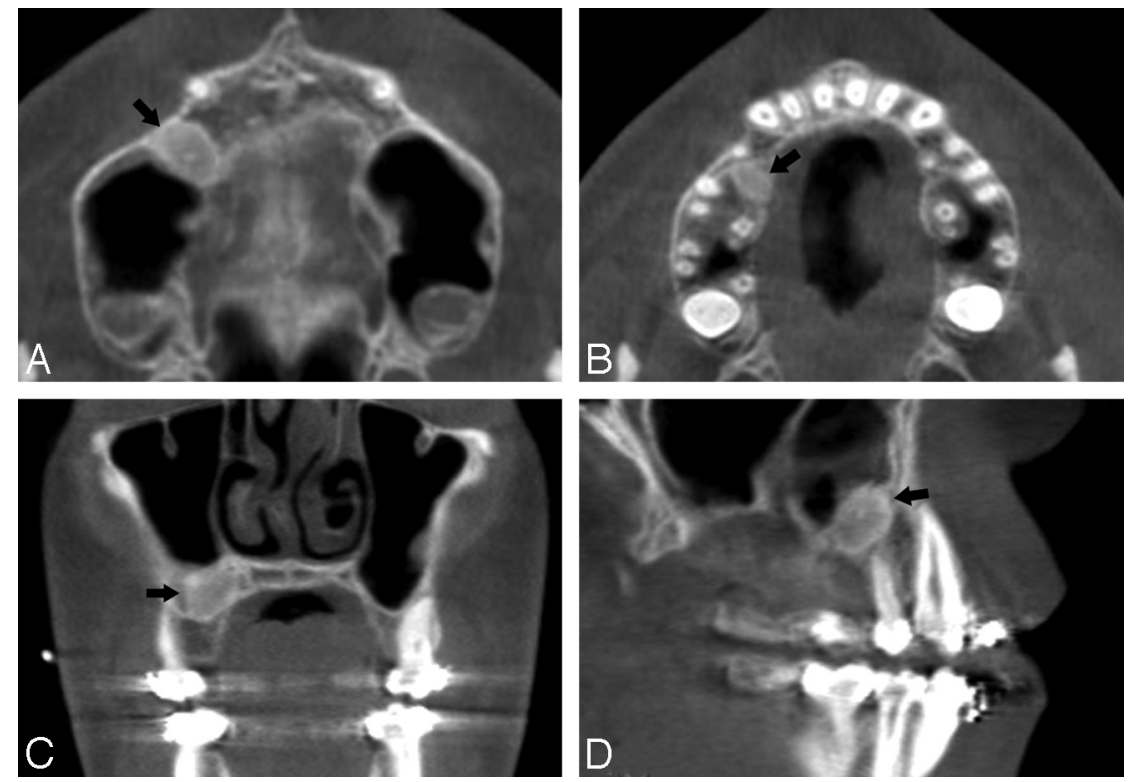

Fig 5. Noncontrast CBCT scan of a 13-year-old boy acquired with a sinus protocol (40 seconds, 600 frames, 0.4-mm pixels, $120 \mathrm{kVp}, 48 \mathrm{~mA}$ ). $A$, Axial section at the level of the maxillary sinus floor demonstrates a $1.0 \times 0.7 \times 1.0-\mathrm{cm}$ oval mass in the alveolar process of the right maxilla (arrow). $B$, Axial image highlights the inferior extent of the lesion in $A$. $C$ and $D$, Coronal and sagittal images, respectively, of the lesion in $A$.

processes such as degenerative changes and ankylosis, joint remodeling after diskectomy, malocclusion, and congenital and developmental malformations. ${ }^{49}$ CBCT is a technique that has recently inspired research in TMJ imaging, though preliminary experiments have yet to translate into clinical studies. Several cadaveric series have explored the use of TMJ CBCT to assess periarticular bony defects, flattenings, osteophytes, and sclerotic changes. ${ }^{50-53}$ Preliminary studies have also directly compared $\mathrm{CBCT}$ with radiography, multidetector row CT (MDCT), and linear tomography for detection of osseous abnormalities of the TMJ. ${ }^{52,53}$ Although early results are promising, more research is needed before CBCT should be used clinically to assess the TMJ. A recent systematic review by Hussain et $\mathrm{al}^{54}$ suggests that axially corrected sagittal tomography is still the method of choice in the detection of periarticular erosions and osteophytes.

\section{Endodontics}

CBCT has been explored for applications in endodontics, including periradicular surgical planning, assessment of periapical pathology, and dentoalveolar trauma evaluation..$^{55}$ The diagnostic properties of $\mathrm{CBCT}$ at the root apices and perira- 

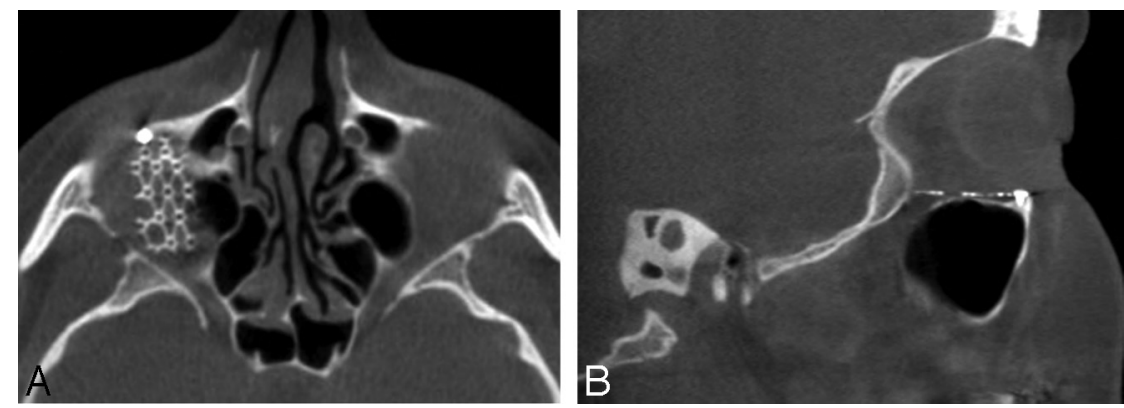

Fig 6. Noncontrast CBCT scan of a 45-year-old ( 40 seconds, 600 frames, $0.4-\mathrm{mm}$ pixels, $120 \mathrm{kVp}, 48 \mathrm{~mA}$ ) obtained after repair of a right orbital floor fracture. $A$, Axial image demonstrates a metal fixture implanted over the right orbital floor. $B$, Metallic implant is seen in the sagittal section.

dicular region have been reported in several studies. ${ }^{56-58}$ In retrospective cohorts and case reports, CBCT has been suggested as superior to periapical radiographs in the characterization of periapical lucent lesions, reliably demonstrating lesion proximity to the maxillary sinus, sinus membrane involvement, and lesion location relative to the mandibular canal. $^{56-58}$ There may also eventually be a role for CBCT in early detection of periapical disease, which could lead to better endodontic treatment outcomes. ${ }^{55}$ Promising results have been demonstrated in studies characterizing CBCT images for endodontic surgical planning purposes as well. ${ }^{58,59}$

\section{Periodontics}

The first reported applications of CBCT in periodontology were for diagnostic and treatment-outcome evaluations of periodontitis. ${ }^{60}$ Ex vivo studies later characterized the ability of CBCT to accurately reconstruct periodontal intrabony and fenestration defects, dehiscences, and root furcation involvements in comparison with radiography, MDCT, and histologic measurements. ${ }^{61-65}$ CBCT 3D geometric accuracy has been suggested to be equal to radiography and MDCT but with better observer-rated image quality than MDCT as well as superior periodontal-defect detection than radiography. ${ }^{61,62,64}$ Although periodontal bony defects are well visualized with CBCT, conventional radiography still affords higher quality bony contrast and delineation of the lamina dura. ${ }^{63}$ CBCT ex vivo visualization of the periodontal ligament and periodontal ligament space has been evaluated in comparison with radiography with mixed results, a more recent study suggesting that CBCT visualization is still inferior to that of radiography. ${ }^{61,66}$

\section{Head and Neck}

As CBCT imaging systems have become more widely available, interest in the intraoperative and diagnostic CBCT applications in the extracranial head and neck regions has intensified. The reported high isotropic spatial resolution and relatively low dose requirements of $\mathrm{CBCT}$ are characteristics that have made it particularly attractive. In the head and neck region, a premium is placed on discriminating fine anatomic detail in territories where the vascular and bony structural anatomy is particularly complex. ${ }^{8}$ Potential applications in sinus, temporal bone, and skull base imaging have been explored, as discussed below. Figures 3, 4, 5, and 6 depict head and neck CBCT studies visualizing the paranasal sinuses; temporal bones; maxillary sinus floor and alveolar process of the maxilla; and orbital floors respectively.

\section{Sinus Imaging/Frontal Recess}

Comparatively low dosing requirements, high-quality bony definition, and the compact design afforded by CBCT scanners have made them attractive for office-based and intraoperative scanning of the paranasal sinuses. ${ }^{67,68}$ To date, there have been few studies comparing image quality in paranasal sinus CBCT scans with that in MDCT. Alspaugh et al ${ }^{69}$ did directly compare the spatial resolution obtained with CBCT scans of the paranasal sinuses with that of 16- and 64-section MDCT scanners. They concluded that 12 line pairs per centimeter $(\mathrm{lp} / \mathrm{cm})$ isotropic spatial resolution could be obtained with an effective dose of $0.17 \mathrm{mSv}$ compared with a dose requirement of $0.87 \mathrm{mSv}$ for $11-\mathrm{lp} / \mathrm{cm}$ spatial resolution in a 64-section MDCT scanner.

To a large degree, evidence supporting sinus CBCT imaging has emerged from exploration of intraoperative CBCT applications in endoscopic sinus surgery (ESS). In preclinical cadaver studies, Rafferty et $\mathrm{al}^{67}$ provided proof of principle for the application of C-arm CBCT imaging to ESS, concluding that both spatial and soft-tissue contrast was sufficient to aid surgical navigation in the frontal recess. More recent clinical studies have also provided qualitative evidence that intraoperative CBCT provides high-quality definition of bony anatomy, which can lead to refinement of surgical strategy. ${ }^{70,71}$ In a series of 25 patients undergoing ESS, Batra et $\mathrm{al}^{71}$ found that residual bony partitions and stent locations could be visualized with intraoperative CBCT scans, leading to surgical revision. CBCT has also been used recently to evaluate contrast delivery during sinus irrigation after ESS. ${ }^{72}$

Preliminary evidence suggests that CBCT may be suited for specific imaging tasks in the context of intraoperative and perioperative bony structural evaluations, enabling low-dose assessment of individualized paranasal sinus anatomy, surgical outcomes, and stent placements. To our knowledge, there is no current evidence, however, supporting CBCT use in general diagnostic sinus imaging owing to lack of soft-tissue contrast resolution. Furthermore, significant complications of ESS, including encephalocele, subarachnoid hemorrhage, and meningitis are unlikely to be evaluated adequately with current CBCT image quality. ${ }^{73,74}$

\section{Temporal Bone/Lateral Skull Base}

The temporal bone was one of the earliest targets for head and neck CBCT imaging. Specific applications have been explored, including postprocedural middle and inner ear implant evaluation, visualization of the reuniting duct in the inner ear, and intraoperative temporal bone surgical guidance. . $^{8,75,76}$ 
Preliminary evaluation of an experimental CBCT system for general temporal bone diagnostic imaging was performed by Gupta et $\mathrm{al}^{12}$ on a small series of partially manipulated cadaveric specimens. They found that observer scores of the quality of structural visualization with CBCT were significantly higher compared with scores for MDCT. Particularly well-visualized structures included the ossicular chain, bony labyrinth of the inner ear, internal cochlear anatomy, and the facial nerve. They also noted reduced metal artifacts with cochlear implant imaging as well as improved detection of small laser-induced lesions in the ossicular chain. Gupta et al suggest that lack of soft-tissue contrast in their evaluations did not interfere with diagnostic accuracy due to the abundance of high-contrast structures housed in the temporal bone and the positive effect of higher spatial resolution on resolving some low-contrast structures such as the facial nerve.

Peltonen et al $^{14}$ compared a commercially available CBCT scanner with MDCT in a study on unoperated temporal bone specimens by using a modified Likert scale (scored by 2 otologists and 1 radiologist) to assess visualization of important structures in the lateral skull base. They concluded that CBCT was at least as accurate as MDCT in defining surgically relevant middle ear structures. The inner ear was incompletely visualized with CBCT in their study.

Perhaps the most well-studied use of temporal bone CBCT is for the evaluation of middle and inner ear implants. Early preclinical studies in temporal bone specimens fitted with cochlear implants demonstrated that an adapted CT angiography CBCT system could noninvasively depict the electrodemodiolus relationship postimplantation. ${ }^{77,78}$ These results were later corroborated in another cadaver study comparing single- and multisection CT with CBCT. ${ }^{79}$ When compared with single- or multisection CT, a reduction in metal artifacts was observed with CBCT, which allowed more precise determination of electrode-array positioning within the scala tympani or scala vestibuli. ${ }^{79}$ Reduced metal artifacts with implant imaging using CBCT compared with conventional CT were also demonstrated by Offergeld et $\mathrm{al}^{80}$ in a study evaluating middle ear implants in postsurgical temporal bone specimens.

Preclinical studies have been followed by studies of patients with inner and middle ear implants, suggesting that the combination of high spatial resolution and reduced metal artifacts with CBCT imaging may facilitate the postsurgical evaluation of reconstructed middle and inner ears. ${ }^{75,81}$ A recent study has also explored the utility of CBCT in evaluating progressive hearing loss. Dalchow et $\mathrm{al}^{82}$ submitted 25 patients with audiometry-confirmed conductive hearing loss to preoperative CBCT and concluded that CBCT could be accurate both in predicting the continuity of the ossicular chain and in detecting ossicular erosions.

Multiple commercial CBCT systems have temporal bone-acquisition protocols. The miniCAT acquires temporal bone images at 125 kilovolt (peak) $(\mathrm{kVp})$ and $58.8 \mathrm{~mA}$ with a 20-second scanning time using a sharp kernel (manufacturer's data). This protocol delivers 4.62 and $4.18 \mathrm{mGy}$ at the center and periphery, respectively, of a $100-\mathrm{mm}$ ion chamber, achieving spatial resolution in the range of $14-16 \mathrm{lp} / \mathrm{cm}$ (manufacturer's data). In their study of limited-FOV temporal bone imaging described above, Peltonen et $\mathrm{al}^{14}$ noted a 60 -fold effective dose reduction with CBCT compared with MDCT, though they attributed much of this dramatic reduction to significantly smaller FOVs and shorter scanning times for their CBCT images. They noted that low-dose MDCT settings can acquire images with effective doses like those in CBCT if the FOVs and scanning times are, in fact, comparable with those of CBCT. ${ }^{14}$

These data suggest that CBCT might be useful for select imaging tasks in temporal bone imaging, including evaluation of inner and middle ear implant positioning, as well as definition of high-contrast postsurgical change and structural anatomy within the lateral skull base. Possible applications in evaluation of bony pathology, such as ossicular chain erosions, may also be emerging. Currently, further research is required to characterize the ability of CBCT to define temporal bone structures and bony pathology reliably, especially given the technologic and scan-parameter variability of commercial CBCT scanners. Lack of soft-tissue contrast resolution also continues to limit the use of CBCT in general diagnostic imaging of the temporal bone.

\section{Skull Base}

The particularly complex bony and neurovascular anatomy of the skull base makes it an attractive target for high-spatialresolution imaging. Current practices in oncologic imaging of the skull base rely on MDCT and MR imaging for combined osseous and soft-tissue definitions. ${ }^{83}$ Several preclinical reports have begun to explore the potential uses of CBCT during surgeries at the skull base, ${ }^{7,84,85}$ suggesting high $3 \mathrm{D}$ localization accuracy and low target-registration error with effective doses in the range of $0.1-0.35 \mathrm{mSv}$. The xCAT intraoperative CBCT scanner (Xoran Technologies), a cousin of the MiniCat, has been evaluated in clinical scenarios at the skull base as well, with favorable preliminary results. ${ }^{71,86}$

\section{Controversies}

As with any emerging imaging technology, use of CBCT scanners has been the subject of criticism as well as acclaim. The technology itself is limited by lack of user experience and what is currently a relatively small body of related literature. The point-of-service operational model that dominates diagnostic head and neck CBCT imaging practices has also drawn criticism. Additionally, the ACR Practice Guideline for CT of the head and neck recommends that all imaging studies be evaluated with bone and soft-tissue algorithms. ${ }^{87}$ Because of the low radiation dose, CBCT can only provide bony detail and is unable to provide images of the soft tissues.

At our institution, CBCT scanning of the extracranial head and neck is performed in both a clinical and research capacity primarily for sinus, maxillofacial, and occasional temporal bone imaging. Research on this technology is still preliminary, without prospective studies that convincingly demonstrate its benefit compared with conventional CT.

Both in medical and oral and maxillofacial imaging in dentistry, CBCT has been largely adopted as an office-based service. This is a usage model purported to expedite patient diagnosis and treatment while simultaneously reducing costs, providing 1-stop management with fewer billed visits and no radiologist consultation fees. Point-of-service imaging and other self-referral services, however, have been widely criti- 
cized for encouraging overuse and directly inflating medical costs.

The belief that financial incentives undermine the clinical decision-making process has been the basis for federal legislation limiting Medicare payments for self-referral services (socalled "Stark laws"). Regulatory checks on the operation of imaging technologies are present in many states in the form of Certificates of Need (CONs), which require documentation of sufficient community demand before a technology can be certified to be operated at a particular facility. This theoretically prevents excess capacity of medical equipment and prevents cost inflation. In states with CON laws, CBCT scanners are often treated like conventional CT scanners and are subject to the same scanning-volume requirements regulating the acquisition and operation of conventional CT scanners. The CT allowance in a given community is typically filled by conventional scanners, making it difficult to operate a CBCT machine in states with CON laws.

The advent of CBCT technologies has also fueled the controversy surrounding office-based imaging, which is usually performed and interpreted by nonradiologists often without the accreditation, training, or licensure afforded by the radiology community. A recent position paper by the American Academy of Oral and Maxillofacial Radiology addressed this issue, emphasizing the role of the practitioner in obtaining and interpreting CBCT images. It highlights the practitioner's responsibility for understanding CBCT operating parameters, reviewing the entire exposed tissue volume, and addressing all radiologic findings irrespective of their association with the scanning indication. ${ }^{88}$

\section{Conclusions}

CBCT is an emerging CT technology, which has potential applications for imaging of high-contrast structures in the head and neck as well as dentomaxillofacial regions. Preliminary research suggests that high-spatial-resolution images can be obtained with comparatively low patient dose. To date, the most researched applications for head and neck CBCT are in sinus, middle and inner ear implant, and dentomaxillofacial imaging. This technology is not without controversy, and further research is required to establish informed recommendations about its appropriate use in a clinical setting.

\section{Acknowledgments}

We thank Karen Carter for her help with image preparation; Sharon Brooks, for providing the images in figures 1 and 2; and Sylvia Miracle, for her review of the manuscript.

\section{References}

1. Robb RA. The dynamic spatial reconstructor: an $\mathrm{x}$-ray video-fluoroscopic CT scanner for dynamic volume imaging of moving organs. IEEE Trans Med Imaging 1982;1:22-33

2. Fahrig R, Fox AJ, Lownie S, et al. Use of a C-arm system to generate true three-dimensional computed rotational angiograms: preliminary in vitro and in vivo results. AJNR Am J Neuroradiol 1997;18:1507-14

3. Saint-Félix D, Trousset $Y$, Picard C, et al. In vivo evaluation of a new system for 3D computerized angiography. Phys Med Biol 1994;39:583-95

4. Silver MD, Yahata M, Saito Y, et al. Volume CT of anthropomorphic phantoms using a radiation therapy simulator. In: Shaw R, ed. Medical Imaging VI: Instrumentation: Proceedings of SPIE 1992;1651:197-211

5. Cho PS, Johnson RH, Griffin TW. Cone-beam CT for radiotherapy applications. Phys Med Biol 1995:40:1863-83

6. Jaffray DA, Drake DG, Moreau M, et al. A radiographic and tomographic im- aging system integrated into a medical linear accelerator for localization of bone and soft-tissue targets. Int J Radiat Oncol Biol Phys 1999;45:773-89

7. Daly MJ, Siewerdsen JH, Moseley DJ, et al. Intraoperative cone-beam CT for guidance of head and neck surgery: assessment of dose and image quality using a C-arm prototype. Med Phys 2006;33:3767-80

8. Rafferty MA, Siewerdsen JH, Chan Y, et al. Intraoperative cone-beam CT for guidance of temporal bone surgery. Otolaryngol Head Neck Surg 2006;134: 801-08

9. Siewerdsen JH, Moseley DJ, Burch S, et al. Volume CT with a flat-panel detector on a mobile, isocentric $\mathrm{C}$-arm: pre-clinical investigation in guidance of minimally invasive surgery. Med Phys 2005;32:241-54

10. Khoury A, Siewerdsen JH, Whyne CM, et al. Intraoperative cone-beam CT for image-guided tibial plateau fracture reduction. Comput Aided Surg 2007;12: 195-207

11. Khoury A, Whyne CM, Daly M, et al. Intraoperative cone-beam CT for correction of periaxial malrotation of the femoral shaft: a surface-matching approach. Med Phys 2007;34:1380-87

12. Gupta R, Bartling SH, Basu SK, et al. Experimental flat-panel high-spatialresolution volume CT of the temporal bone. AJNR Am J Neuroradiol 2004;25: 1417-24

13. Chen B, Ning R. Cone-beam volume CT breast imaging: feasibility study. Med Phys 2002;29:755-70

14. Peltonen LI, Aarnisalo AA, Kortesniemi MK, et al. Limited cone-beam computed tomography imaging of the middle ear: a comparison with multislice helical computed tomography. Acta Radiol 2007;48:207-12

15. Orth RC, Wallace MJ, Kuo MD, for the Technology Assessment Committee of the Society of Interventional Radiology. C-arm cone-beam CT: general principles and technical considerations for use in interventional radiology. $J$ Vasc Interv Radiol 2008;19:814-20. Epub 2008 Apr 23

16. Dörfler A, Struffert T, Engelhorn T, et al. Rotational flat-panel computed tomography in diagnostic and interventional neuroradiology. Rofo 2008;180: 891-98

17. Moore CJ, Am A, Marchant T, et al. Developments in and experience of kilovoltage X-ray cone beam image-guided radiotherapy. Br J Radiol 2006;79(Spec No 1):S66-78

18. Scarfe WC, Farman AG, Sukovic P. Clinical applications of cone-beam computed tomography in dental practice. J Can Dent Assoc 2006;72:75-80

19. Glick SJ. Breast CT. Annu Rev Biomed Eng 2007;9:501-26

20. Mozzo P, Procacci C, Tacconi A, et al. A new volumetric CT machine for dental imaging based on the cone-beam technique: preliminary results. Eur Radiol 1998;8:1558-64

21. Arai Y, Tammisalo E, Iwai K, et al. Development of a compact computed tomographic apparatus for dental use. Dentomaxillofac Radiol 1999;28:245-48

22. Lascala CA, Panella J, Marques MM. Analysis of the accuracy of linear measurements obtained by cone beam computed tomography (CBCT-NewTom). Dentomaxillofac Radiol 2004;33:291-94

23. Mischkowski RA, Pulsfort R, Ritter L, et al. Geometric accuracy of a newly developed cone-beam device for maxillofacial imaging. Oral Surg Oral Med Oral Pathol Oral Radiol Endod 2007;104:551-59. Epub 2007 Jul 5

24. Lagravère MO, Carey J, Toogood RW, et al. Three-dimensional accuracy of measurements made with software on cone-beam computed tomography images. Am J Orthod Dentofacial Orthop 2008;134:112-16

25. Kumar V, Ludlow JB, Mol A, et al. Comparison of conventional and cone beam CT synthesized cephalograms. Dentomaxillofac Radiol 2007;36:263-69

26. Ludlow JB, Ivanovic M. Comparative dosimetry of dental CBCT devices and 64-slice CT for oral and maxillofacial radiology. Oral Surg Oral Med Oral Pathol Oral Radiol Endod 2008;106:106-14. Epub 2008 May 27

27. Schulze D, Heiland $M$, Thurmann $H$, et al. Radiation exposure during midfacial imaging using 4- and 16-slice computed tomography, cone beam computed tomography systems and conventional radiography. Dentomaxillofac Radiol 2004;33:83-86

28. Tyndall DA, Brooks SL. Selection criteria for dental implant site imaging: a position paper of the American Academy of Oral and Maxillofacial Radiology. Oral Surg Oral Med Oral Pathol Oral Radiol Endod 2000;89:630-37

29. Lofthag-Hansen S, Gröndahl K, Ekestubbe A. Cone-beam CT for preoperative implant planning in the posterior mandible: visibility of anatomic landmarks. Clin Implant Dent Relat Res 2008 Sep 9. [Epub ahead of print]

30. Guerrero ME, Jacobs R, Loubele M, et al. State-of-the-art on cone beam CT imaging for preoperative planning of implant placement. Clinical Oral Investig 2006;10:1-7

31. Ito K, Gomi Y, Sato S, et al. Clinical application of a new compact CT system to assess 3-D images for the preoperative treatment planning of implants in the posterior mandible: a case report. Clin Oral Implants Res 2001;12:539-42

32. Sato S, Arai Y, Shinoda K, et al. Clinical application of a new cone-beam computerized tomography system to assess multiple two-dimensional images for the preoperative treatment planning of maxillary implants: case reports. Quintessence Int 2004;35:525-28

33. Terakado M, Hashimoto K, Arai Y, et al. Diagnostic imaging with newly developed ortho cubic super-high resolution computed tomography (Ortho-CT). Oral Surg Oral Med Oral Pathol Oral Radiol Endod 2000;89:509-18

34. Ziegler CM, Woertche R, Brief J, et al. Clinical indications for digital volume 
tomography in oral and maxillofacial surgery. Dentomaxillofac Radiol 2002;31:126-30

35. Drage NA, Sivarajasingam V. The use of cone beam computed tomography in the management of isolated orbital floor fractures. Br J Oral Maxillofac Surg 2009;47:65-66. Epub 2008 Jun 30

36. Zizelmann C, Gellrich NC, Metzger MC, et al. Computer-assisted reconstruction of orbital floor based on cone beam tomography. Br J Oral Maxillofac Surg 2007;45:79-80. Epub 2005 Aug 10

37. Heiland M, Schulze D, Blake F, et al. Intraoperative imaging of zygomaticomaxillary complex fractures using a 3D C-arm system. Int J Oral Maxillofac Surg 2005;34:369-75

38. Pohlenz P, Blessmann M, Blake F, et al. Clinical indications and perspectives for intraoperative cone-beam computed tomography in oral and maxillofacial surgery. Oral Surg Oral Med Oral Pathol Oral Radiol Endod 2007;103:41217. Epub 2006 Sep 26

39. Heiland M, Schulze D, Rother U, et al. Postoperative imaging of zygomaticomaxillary complex fractures using digital volume tomography. J Oral Maxillofac Surg 2004;62:1387-91

40. Pohlenz $\mathrm{P}$, Blessmann $\mathrm{M}$, Blake F, et al. Major mandibular surgical procedures as an indication for intraoperative imaging. J Oral Maxillofac Surg 2008;66:324-29

41. Holberg C, Steinhauser S, Geis P, et al. Cone-beam computed tomography in orthodontics: benefits and limitations [in English, German]. J Orofac Orthop 2005;66:434-44

42. Gracco A, Lombardo L, Cozzani M, et al. Quantitative cone-beam computed tomography evaluation of palatal bone thickness for orthodontic miniscrew placement. Am J Orthod Dentofacial Orthop 2008;134:361-69

43. King KS, Lam EW, Faulkner MG, et al. Vertical bone volume in the paramedian palate of adolescents: a computed tomography study. Am J Orthod Dentofacial Orthop 2007;132:783-88

44. Garrett BJ, Caruso JM, Rungcharassaeng K, et al. Skeletal effects to the maxilla after rapid maxillary expansion assessed with cone-beam computed tomography. Am J Orthod Dentofacial Orthop 2008;134:8 -9

45. Yang F, Jacobs R, Willems G. Dental age estimation through volume matching of teeth imaged by cone-beam CT. Forensic Sci Int 2006;159(suppl 1):S78-83. Epub 2006 Mar 23

46. Aboudara CA, Hatcher D, Nielsen IL, et al. A three-dimensional evaluation of the upper airway in adolescents. Orthod Craniofac Res 2003;6(suppl 1):173-75

47. Nakajima A, Sameshima GT, Arai Y, et al. Two- and three-dimensional orthodontic imaging using limited cone beam-computed tomography. Angle Orthod 2005;75:895-903

48. Silva MA, Wolf U, Heinicke F, et al. Cone-beam computed tomography for routine orthodontic treatment planning: a radiation dose evaluation. Am J Orthod Dentofacial Orthop 2008;133:640.e1-5

49. Brooks SL, Brand JW, Gibbs SJ, et al. Imaging of the temporomandibular joint: a position paper of the American Academy of Oral and Maxillofacial Radiology. Oral Surg Oral Med Oral Pathol Oral Radiol Endod 1997;83:609-18

50. Hintze $\mathrm{H}$, Wiese $\mathrm{M}$, Wenzel A. Cone beam CT and conventional tomography for the detection of morphological temporomandibular joint changes. Dentomaxillofac Radiol 2007;36:192-97

51. Honda K, Arai Y, Kashima M, et al. Evaluation of the usefulness of the limited cone-beam CT (3DX) in the assessment of the thickness of the roof of the glenoid fossa of the temporomandibular joint. Dentomaxillofac Radiol 2004;33:391-95

52. Honda K, Larheim TA, Maruhashi K, et al. Osseous abnormalities of the mandibular condyle: diagnostic reliability of cone beam computed tomography compared with helical computed tomography based on an autopsy material. Dentomaxillofac Radiol 2006;35:152-57

53. Honey OB, Scarfe WC, Hilgers MJ, et al. Accuracy of cone-beam computed tomography imaging of the temporomandibular joint: comparisons with panoramic radiology and linear tomography. Am J Orthod Dentofacial Orthop 2007;132:429-38

54. Hussain AM, Packota G, Major PW, et al. Role of different imaging modalities in assessment of temporomandibular joint erosions and osteophytes: a systematic review. Dentomaxillofac Radiol 2008;37:63-71

55. Patel S, Dawood A, Ford TP, et al. The potential applications of cone beam computed tomography in the management of endodontic problems. Int Endod J 2007;40:818-30. Epub 2007 Aug 14

56. Lofthag-Hansen S, Huumonen S, Gröndahl K, et al. Limited cone-beam CT and intraoral radiography for the diagnosis of periapical pathology. Oral Surg Oral Med Oral Pathol Oral Radiol Endod 2007;103:114-19. Epub 2006 Apr 24

57. Nakata K, Naitoh M, Izumi M, et al. Effectiveness of dental computed tomography in diagnostic imaging of periradicular lesion of each root of a multirooted tooth: a case report. J Endod 2006;32:583-87

58. Low KM, Dula K, Bürgin W, von Arx T. Comparison of periapical radiography and limited cone-beam tomography in posterior maxillary teeth referred for apical surgery. J Endod 2008;34:557-62

59. Rigolone M, Pasqualini D, Bianchi L, et al. Vestibular surgical access to the palatine root of the superior first molar: "low-dose cone-beam" CT analysis of the pathway and its anatomic variations. J Endod 2003;29:773-35

60. Ito K, Yoshinuma N, Goke E, et al. Clinical application of a new compact computed tomography system for evaluating the outcome of regenerative therapy: a case report. J Periodontol 2001;72:696-702
61. Mengel R, Candir M, Shiratori K, et al. Digital volume tomography in the diagnosis of periodontal defects: an in vitro study on native pig and human mandibles. J Periodontol 2005;76:665-73

62. Misch KA, Yi ES, Sarment DP. Accuracy of cone beam computed tomography for periodontal defect measurements. J Periodontol 2006;77:1261-66

63. Vandenberghe B, Jacobs R, Yang J. Diagnostic validity (or acuity) of 2D CCD versus 3D CBCT-images for assessing periodontal breakdown. Oral Surg Oral Med Oral Pathol Oral Radiol Endod 2007;104:395-401. Epub 2007 Jul 5

64. Mol A, Balasundaram A. In vitro cone beam computed tomography imaging of periodontal bone. Dentomaxillofac Radiol 2008;37:319-24

65. Vandenberghe B, Jacobs R, Yang J. Detection of periodontal bone loss using digital intraoral and cone beam computed tomography images: an in vitro assessment of bony and/or infrabony defects. Dentomaxillofac Radiol 2008;37: $252-60$

66. Ozmeric N, Kostioutchenko I, Hagler G, et al. Cone-beam computed tomography in assessment of periodontal ligament space: in vitro study on artificial tooth model. Clin Oral Investig 2008;12:233-39. Epub 2008 Feb 5

67. Rafferty MA, Siewerdsen JH, Chan Y, et al. Investigation of C-arm cone-beam CT-guided surgery of the frontal recess. Laryngoscope 2005;115:2138-43

68. Chennupati SK, Woodworth BA, Palmer JN, et al. Intraoperative IGS/CT updates for complex endoscopic frontal sinus surgery. ORL J Otorhinolaryngol Relat Spec 2008;70:268-70. Epub 2008 May 19

69. Alspaugh J, Christodoulou E, Goodsitt M, et al. Dose and image quality of flat-panel detector volume computed tomography for sinus imaging. In: Proceedings of the 49th Annual Meeting of the American Association of Physics in Medicine, Minneapolis, Minn. July 22-26, 2007

70. Jackman AH, Palmer JN, Chiu AG, et al. Use of intraoperative CT scanning in endoscopic sinus surgery: a preliminary report. Am J Rhinol 2008;22:170-74

71. Batra PS, Kanowitz SJ, Citardi MJ. Clinical utility of intraoperative volume computed tomography scanner for endoscopic sinonasal and skull base procedures. Am J Rhinol 2008;22:511-15

72. Harvey RJ, Goddard JC, Wise SK, et al. Effects of endoscopic sinus surgery and delivery device on cadaver sinus irrigation. Otolaryngol Head Neck Surg 2008; 139:137-42

73. Hudgins PA, Browning DG, Gallups J, et al. Endoscopic paranasal sinus surgery: radiographic evaluation of severe complications. AJNR Am J Neuroradiol 1992;13:1161-67

74. Zinreich SJ. Functional anatomy and computed tomography imaging of the paranasal sinuses. Am J Med Sci 1998;316:2-12

75. Cerini R, Faccioli N, Barillari M, et al. Bionic ear imaging. Radiol Med 2008;113 265-77. Epub 2008 Apr 2

76. Yamane H, Takayama M, Sunami K, et al. Three-dimensional images of the reuniting duct using cone beam CT. Acta Otolaryngol 2009;129:493-96

77. Husstedt HW, Aschendorff A, Richter B, et al. Nondestructive three-dimensional analysis of electrode to modiolus proximity. Otol Neurotol 2002;23: $49-52$

78. Richter B, Aschendorff A, Lohnstein P, et al. The Nucleus Contour electrode array: a radiological and histological study. Laryngoscope 2001;111:508-14

79. Aschendorff A, Kubalek R, Hochmuth A, et al. Imaging procedures in cochlear implant patients: evaluation of different radiological techniques. Acta Otolaryngol Suppl 2004;552:46-49

80. Offergeld C, Kromeier J, Aschendorff A, et al. Rotational tomography of the normal and reconstructed middle ear in temporal bones: an experimental study. Eur Arch Otorhinolaryngol 2007;264:345-51. Epub 2006 Oct 18

81. Aschendorff A, Kubalek R, Turowski B, et al. Quality control after cochlear implant surgery by means of rotational tomography. Otol Neurotol 2005;26:34-47

82. Dalchow CV, Weber AL, Bien S, et al. Value of digital volume tomography in patients with conductive hearing loss. Eur Arch Otorhinolaryngol 2006;263:9299. Epub 2005 Sep 15

83. Chong V. The skull base in oncologic imaging. Cancer Imaging 2004;4:5-6

84. Bachar G, Siewerdsen JH, Daly MJ, et al. Image quality and localization accuracy in C-arm tomosynthesis-guided head and neck surgery. Med Phys 2007;34:4664-77

85. Bartling SH, Leinung M, Graute J, et al. Increase of accuracy in intraoperative navigation through high-resolution flat-panel volume computed tomography: experimental comparison with multislice computed tomography-based navigation. Otol Neurotol 2007;28:129-34

86. Woodworth BA, Chiu AG, Cohen NA, et al. Real-time computed tomography image update for endoscopic skull base surgery. J Laryngol Otol 2008;122:36165. Epub 2007 Aug 16

87. Mukherji SK, Gujar S, Jordan JE, et al. ACR Practice Guideline for the Performance of Computed Tomography $(\mathrm{CT})$ of the Extracranial Head and Neck in Adults and Children. ACR Practice Guideline 2006 (Res 12,17,35). Available at: http://www.acr.org/SecondaryMainMenuCategories/quality_safety/guidelines/ dx/head-neck/ct_head_neck.aspx. Accessed January 15, 2009

88. Carter L, Farman AG, Geist J, et al. American Academy of Oral and Maxillofacial Radiology executive opinion statement on performing and interpreting diagnostic cone beam computed tomography. Oral Surg Oral Med Oral Pathol Oral Radiol Endod 2008;106:561-62 